\title{
Sensibilité à la vitesse de déformation des matériaux composites unidirectionnels
}

\section{Applications au verre E/époxy à $60 \%$ de fibres}

\section{Patrick Rozycki - Daniel Coutellier}

\author{
LAMIH, UMR CNRS 8530 \\ Université de Valenciennes et du Hainaut-Cambrésis \\ Le Mont Houy, F-59313 Valenciennes Cedex 9 \\ patrick.rozycki@univ-valenciennes.fr \\ daniel.coutellier@univ-valenciennes.fr
}

RÉSUMÉ. Des essais expérimentaux de traction et de compression sur des stratifiés d'orientations spécifiques ont démontré une dépendance importante vis-à-vis de la vitesse de déformation tant au niveau des caractéristiques matériaux qu'au niveau du type de comportement. Cet article propose donc l'intégration théorique au sein d'une loi élastoplastique endommageable de cette sensibilité à la vitesse de déformation en utilisant une approche basée sur la définition des fluides visqueux. L'ensemble des développements théoriques est ensuite implémenté dans un élément fini de type coque au sein d'un code de calcul explicite. Les simulations numériques des essais expérimentaux de traction et de compression démontrent la validité et la fiabilité de l'approche développée. D'autres essais, basés notamment sur des impacts linéiques et ponctuels de plaques permettent d'aboutir à des conclusions similaires et nous permettent d'entrevoir les perspectives futures à ces travaux.

ABSTRACT. Some experimental tensile and compressive tests, which have been performed for laminates of specific orientations, have displayed a strong dependence with regard to the strain rate as well for the material characteristics as for the behaviour type. Thus this paper proposes the theoretical integration of this strain rate sensitivity within an elastic plastic damaging law. The approach used is based on the definition of viscous fluids. All the theoretical developments are then implemented in a shell finite element within an explicit calculation code. The numerical simulations of experimental tensile and compressive tests show the validity and the reliability of the presented approach. Other tests, which concern linear and punctual impacts of composite plates, allow to lead to similar conclusions and to foresee the future prospects of this work.

MOTS-CLÉS : composites unidirectionnels, loi de comportement, vitesse de déformation, élément fini, impact.

KEYWORDS: unidirectional composites, behaviour law, strain rate, finite element, impact. 


\section{Introduction}

Dans le domaine des transports, l'utilisation des outils de prédiction virtuelle devient de plus en plus courante, en effet elle permet essentiellement de pallier les investissements, parfois lourds, requis par les campagnes expérimentales de certification. Il est donc nécessaire d'affiner les connaissances à propos du comportement des matériaux afin de fournir des outils encore plus fiables et performants. Dans cette optique, notre problématique s'est portée sur l'estimation $\mathrm{du}$ rôle de la vitesse de déformation sur le comportement de composites unidirectionnels, et plus spécialement de composites verre E/époxy à $60 \%$ de fibres. L'objectif final était d'intégrer ce facteur au sein d'un élément fini d'un code de calcul explicite (Pam Crash $\left.{ }^{\mathrm{TM}}, 2000\right)$.

\section{Présentation de l'élément fini}

Cet élément fini de type coque possède entre autres un modèle dit «global » (Gauthier, 1996), (Coutellier et al., 1998), (Coutellier et al., 2000), (Rozycki, 2000). Le comportement d'un pli élémentaire est décrit par une loi homogénéisée qui est basée principalement sur les travaux menés, notamment par P. Ladevèze, au Laboratoire de Mécanique et Technologie de l'ENS Cachan (Gilletta, 1985), (Ladevèze, 1986), (Le Dantec, 1989), (Allix et al., 1990). Cette loi permet de transcrire bon nombre de phénomènes tels l'élasticité, la plasticité et certains types de dégradation, comme la fissuration de la matrice parallèlement aux fibres, la décohésion fibres/matrice et enfin la rupture des fibres (figure 1). L'écoulement plastique est quant à lui pris en compte à l'aide d'un critère de plasticité de type von Mises généralisé, de la notion de contraintes effectives et enfin d'une loi d'écrouissage isotrope. L'ensemble des paramètres matériaux correspondant à cette loi élasto-plastique endommageable est identifié grâce à des tractions ou des compressions simples ou cycliques sur cinq stratifiés d'orientation prédéfinie.

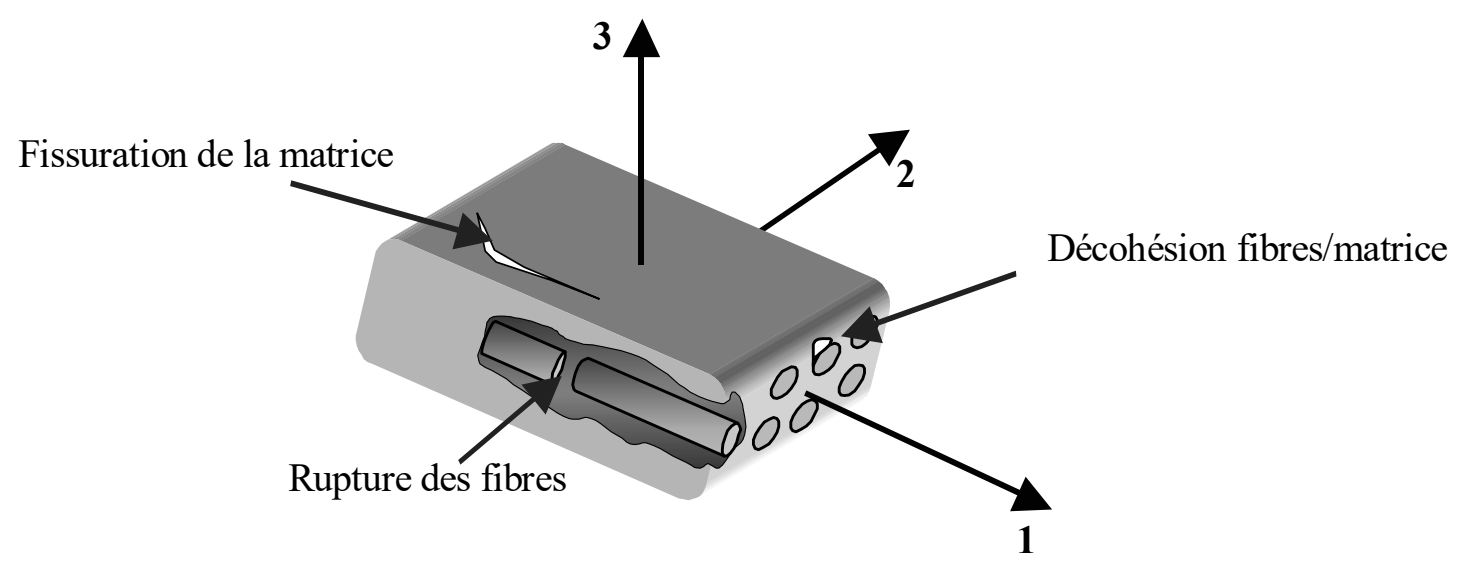

Figure 1. Modes d'endommagement au sein de la couche élémentaire 


\section{Etudes expérimentales}

\subsection{Présentation de la campagne engagée}

Dans un souci constant d'une certaine reproductibilité et faisabilité des tests de caractérisation pour les utilisateurs du code de calcul et afin de conserver l'idéologie théorique du modèle global, nous avons opté pour l'étude de la sensibilité à la vitesse de déformation des paramètres matériaux de la loi élasto-plastique endommageable. Les essais pris en compte sont par conséquent des tractions sur des stratifiés $[0]_{4},[ \pm 45]_{S},[ \pm 67.5]_{S}$ et $[+45]_{4}$ et enfin une compression sur un stratifié $[0]_{6}$.

Le dispositif expérimental est constitué d'une machine de traction/compression dynamique (vérin hydraulique). Pour chaque type d'essai, quatre vitesses de sollicitation ont été principalement appliquées : $5 \mathrm{~mm} / \mathrm{min}, 500 \mathrm{~mm} / \mathrm{min}, 0.2 \mathrm{~m} / \mathrm{s}$ et $4 \mathrm{~m} / \mathrm{s}$. Quelques vitesses intermédiaires ont été introduites, augmentant ainsi l'acuité des résultats. La gamme de vitesse de déformation couverte se situe du quasistatique à des vitesses de déformation de l'ordre de $150 \mathrm{~s}^{-1}$.

\subsection{Observations expérimentales}

Tout comme pour l'identification des paramètres du modèle «quasi-statique », l'ensemble de ces essais permet d'accéder aux comportements dans les directions longitudinales, transversales et de cisaillement.

Deux conclusions essentielles émanent des essais expérimentaux : comme le montrent les figures 2 et 3 , les modules élastiques, la contrainte initiale d'écoulement plastique et enfin la contrainte à rupture longitudinale subissent un accroissement significatif à partir d'un certain seuil de vitesse de déformation. Ces accroissements sont de l'ordre de $30 \%$ à $300 \%$ suivant les paramètres. Enfin, sur la figure 2, nous constatons dans le cas longitudinal qu'il existe une conservation du type de comportement puisque celui-ci demeure élastique fragile. Cependant, dans les deux autres cas - dont celui en cisaillement de la figure 3 - le type de comportement tend à devenir «élastique endommageable » sous l'influence de la vitesse de déformation.

Par conséquent, la loi de comportement peut être qualifiée globalement de loi élasto-plastique endommageable "visqueuse» et peut être, à une vitesse de déformation donnée, toujours identifiée à partir d'une loi de comportement «de référence ». En choisissant pour référence le cas quasi-statique et en utilisant les équations [1] et [2] (Rozycki, 2000), les évolutions de l'ensemble des paramètres précédents sont, pour ce composite verre E/époxy, d'une forme analytique puissance en fonction de la vitesse de déformation [3] (figure 4). Les coefficients des diverses lois sont regroupés dans le tableau 1. 
$X_{\text {courant }}=X_{\text {réference }}[1+f(\underset{\text { courant }}{\&}, \underset{\text { reférence }}{\&})]$ avec X : paramètres élastiques

$X_{\text {normalisé-rapportée }}=\frac{X_{\text {courant }}}{X_{\text {référence }}}-1=X_{\text {normalisé }}-1 \quad$ avec $\quad X_{\text {normalisée }}=\frac{X_{\text {courant }}}{X_{\text {référence }}}$

$f($ courant, référence $)=D\left(\frac{\text { courant }}{\text { référence }}\right)^{m}$

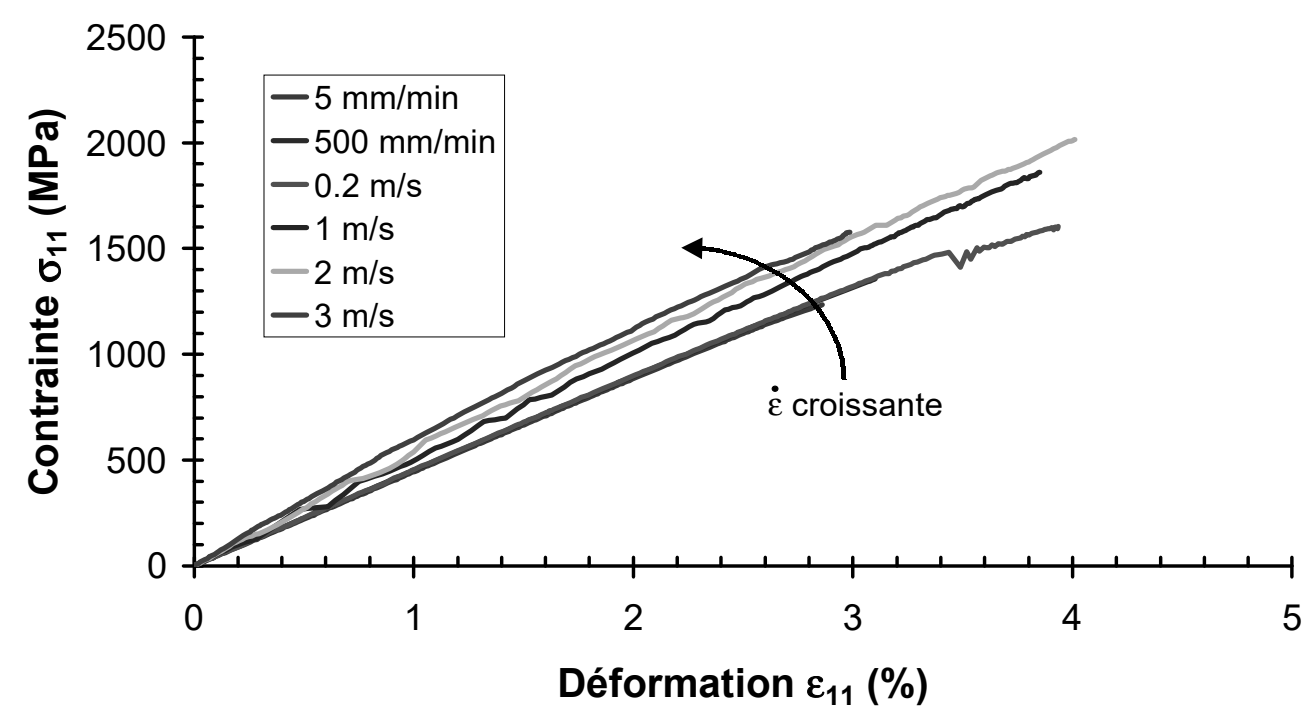

Figure 2. Tractions dynamiques sur un stratifié $[0]_{4}$

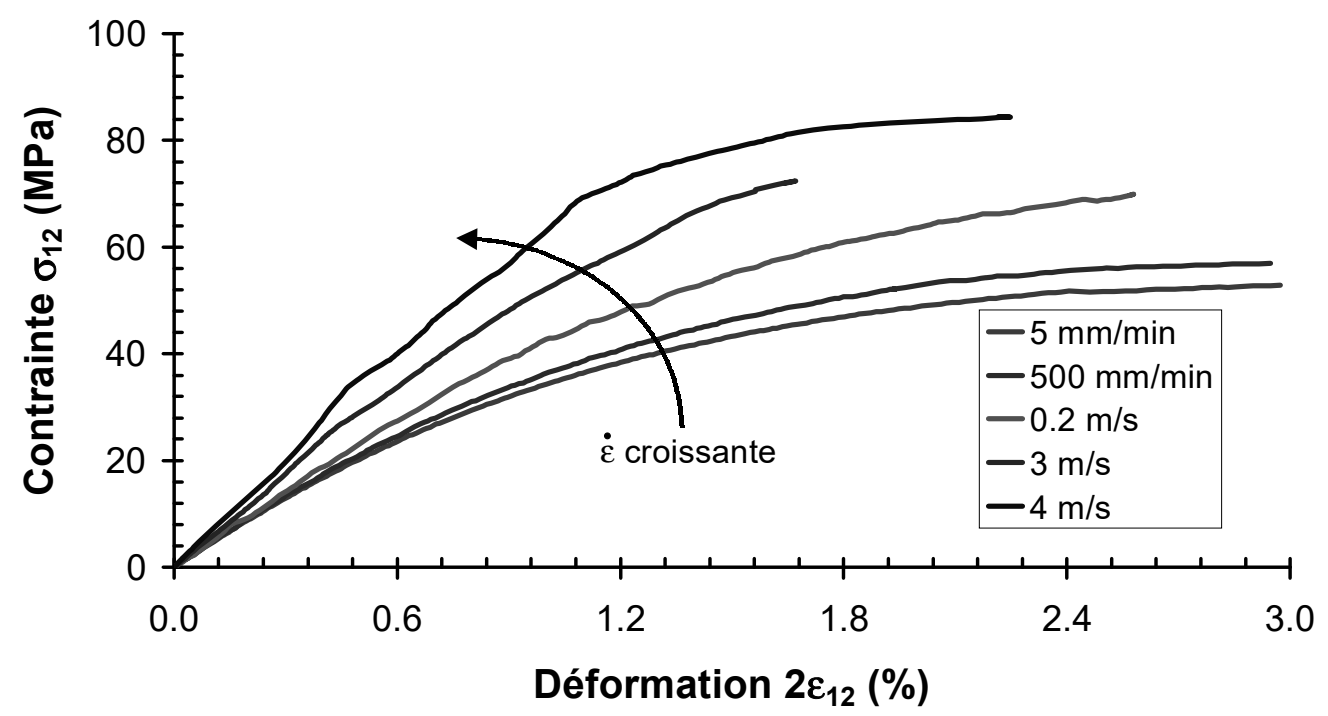

Figure 3. Tractions dynamiques sur un stratifié $[ \pm 45]_{S}$ 


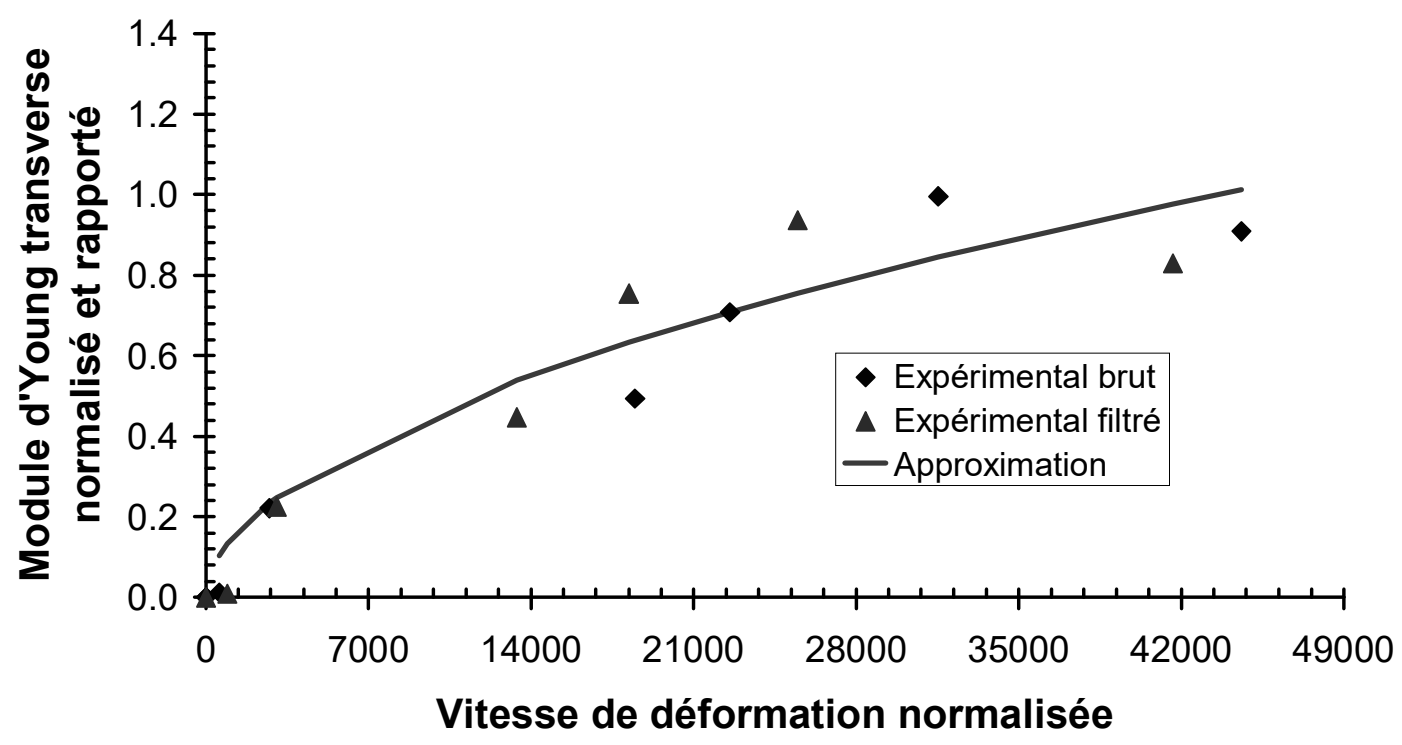

Figure 4. Identification de la loi d'évolution pour le module d'Young transversal

\begin{tabular}{|l|c|c|c|c|}
\hline Tests & Paramètres & $\mathbf{D}(-)$ & $\mathbf{m}(-)$ & \& $_{\text {reference }}^{\text {(-) }}$ \\
\hline \multirow{2}{*}{ Traction sur $[0]_{4}$} & $E_{11}^{0}$ & $1.6110^{-3}$ & $4.5110^{-1}$ & $5.5410^{-4}$ \\
\cline { 2 - 5 } & $\mathcal{E}_{11-U}^{T}$ & $5.6210^{-2}$ & $1.8310^{-1}$ & $5.5410^{-4}$ \\
\hline \multirow{2}{*}{ Traction sur $[ \pm 45]_{S}$} & $G_{12}^{0}$ & $1.8110^{-3}$ & $5.4810^{-1}$ & $9.0810^{-4}$ \\
\cline { 2 - 5 } & $R_{0}$ & $1.3210^{-2}$ & $5.3210^{-1}$ & $9.0810^{-4}$ \\
\hline Traction sur $[+45]_{4}$ & $E_{22}^{0}$ & $3.8610^{-3}$ & $5.3110^{-1}$ & $1.0310^{-3}$ \\
\hline \multirow{2}{*}{ Compression sur $[0]_{6}$} & $E_{11}^{0-C}$ & $1.4210^{-3}$ & $4.6210^{-1}$ & $5.2510^{-4}$ \\
\cline { 2 - 5 } & $\varepsilon_{11-U}^{C}$ & - & - & - \\
\hline
\end{tabular}

Tableau 1. Coefficients des lois d'évolution pour les essais dynamiques

\section{Modélisation théorique visqueuse appliquée}

\subsection{Dépouillement théorique des résultats}

Le type de comportement observé expérimentalement est difficilement identifiable: il ne s'agit, à proprement parler, ni de viscoélasticité, ni de viscoplasticité. Cependant, il est possible de se rapprocher de la définition proposée par Lemaître et Chaboche en ce qui concerne les fluides visqueux (Lemaître, 1985).

En effet, les résines époxy, et plus généralement les polymères réticulés, sont sensibles à la vitesse de déformation au cours des sollicitations (Chaboche, 1995) : 
la viscosité provient essentiellement du mouvement des segments de chaînes au travers des barrières de potentiels énergétiques. Ces phénomènes sont d'autant plus actifs (puisqu'ils sont thermiquement activés) que la température de transition vitreuse de la résine époxy est relativement basse (entre $50{ }^{\circ} \mathrm{C}$ et $150{ }^{\circ} \mathrm{C}$ ). Nous pouvons être amenés à penser que cette élévation de la température, fonction de la vitesse de déformation, impose des micro-changements de phase à la résine qui tend à devenir ainsi un corps «mou ».

Cependant, remarquons que cette influence sur le comportement du matériau est certainement liée à la dépendance de la résine vis-à-vis de cette vitesse de déformation, mais est aussi étroitement liée au choix des constituants. Des travaux sur des stratifiés unidirectionnels carbone/époxy (Allix et al., 1999) montrent une insensibilité des modules élastiques par rapport à la vitesse de déformation : les fibres de carbone possèdent un module d'Young en moyenne trois fois plus élevé que celui des fibres de verre $\mathrm{E}$ et inhibent ainsi les effets visqueux de la résine dans le domaine élastique.

\subsection{Développements théoriques}

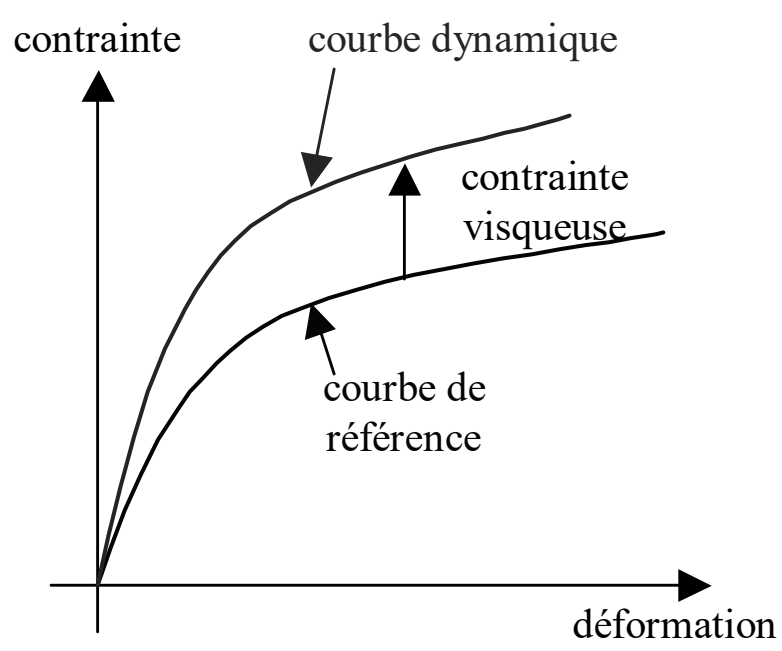

Figure 5. Définition de la contrainte visqueuse

La «pseudo-viscoélasticité » de la résine, et en l'occurrence du composite stratifié (Rozycki, 2000), est prise en compte à l'aide d'une contrainte visqueuse qui vient s'ajouter à la contrainte élastique [4]. En effet, nous avons vu qu'expérimentalement il était toujours possible d'obtenir le comportement à une vitesse donnée en fonction d'un comportement à une vitesse de référence (figure 5).

$\sigma=\sigma^{e}+\sigma^{v}$ 
Cette partition de contrainte n'est pas sans rappeler le modèle de solide de Kelvin-Voigt. Par conséquent, afin d'intégrer le caractère pseudo-viscoélastique, l'idée est de considérer, tout comme dans la théorie viscoélastique de ce type de solide, que la forme du potentiel de dissipation est identique à celle du potentiel thermodynamique. L'énergie de déformation élastique s'écrit donc moyennant des fonctions caractéristiques de la viscosité de la résine [5]. Le tenseur des contraintes s'écrit dès lors, d'après la loi d'état et la loi complémentaire, suivant l'équation [6].

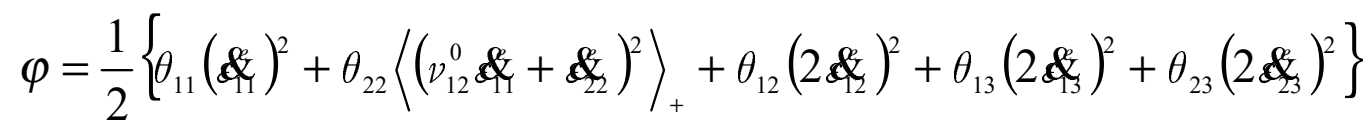

$$
\begin{aligned}
& \stackrel{\sigma}{\underline{\sigma}}=\bar{C}^{0}: \stackrel{e}{\underline{\varepsilon}}+\bar{\Theta}: \stackrel{d e}{=}
\end{aligned}
$$

Dans la direction longitudinale, la relation découlant de [6] est donnée par [7]. Cette dernière équation différentielle (par rapport au temps) possède [8] pour solution si l'on considère qu'à l'état initial, la déformation longitudinale est nulle. Les temps lors des sollicitations dynamiques (crash, impact, etc.) sont extrêmement faibles ; l'expression [8] peut être développée au premier ordre selon [9].

$$
\begin{aligned}
& \sigma_{11}=E_{11}^{0} \varepsilon_{11}^{e}+\theta_{11} \& \\
& \varepsilon_{11}^{e}=\frac{\sigma_{11}}{E_{11}^{0}}\left(1+e^{-\frac{E_{11}^{0}}{\theta_{11}}}\right) \\
& \varepsilon_{11}^{e} \approx \frac{\sigma_{11}}{E_{11}^{0}}\left(1-\frac{E_{11}^{0}}{\theta_{11}} t\right) \Rightarrow \sigma_{11} \approx E_{11}^{0} \varepsilon_{11}^{e}\left(2+\frac{E_{11}^{0}}{\theta_{11}} t\right) \approx E_{11}^{0} \varepsilon_{11}^{e}\left(1+F_{11}\right)
\end{aligned}
$$

Puisque le terme de l'exponentielle est sans dimension, cette équation [9] permet de considérer une évolution du module élastique à l'aide d'une fonction $F_{11}$ qui, dans notre cadre d'étude, peut dépendre de la vitesse de déformation. Si l'on applique ce schéma à chacune des directions, on détermine ainsi chacune des fonctions de viscosité $F$ décrivant l'évolution des modules d'élasticité en fonction de la vitesse de déformation. La relation élastique s'écrit synthétiquement [10] :

$$
=C^{0}:{ }^{e}+:_{=}^{e}=C^{0}(I+F):{ }_{=}^{e}
$$

Nous n'avons pour l'instant qu'une modélisation élastique du comportement dépendante de la vitesse de déformation. Il est par conséquent nécessaire d'adjoindre l'endommagement et la plasticité. En préambule, rappelons que le comportement de la résine est élasto-plastique endommageable, identique à celui qui a été exposé précédemment pour le composite puisqu'il provient d'une homogénéisation de la couche élémentaire. On retrouve par conséquent deux 
variables d'endommagement (pour les cas transverses et de cisaillement) et une évolution du domaine de plasticité de type écrouissage isotrope.

Phénoménologiquement, l'état du matériau (résine ou composite) peut être toujours déterminé à partir de son état dans la configuration quasi-statique. On peut donc supposer que l'état pour une vitesse de déformation donnée correspond à l'état quasi-statique d'un autre matériau composite verre E/époxy dont les caractéristiques matériaux sont différentes. Puisque les modules élastiques n'évoluent que de façon croissante avec la vitesse de déformation, cette hypothèse conduit à postuler une progression de l'endommagement plus rapide que dans le cas statique. Elle laisse présupposer aussi que le domaine de plasticité se déplace de façon conséquente (avec une évolution de la contrainte d'écoulement initiale). Expérimentalement, on constate effectivement une évolution $\mathrm{du}$ comportement élasto-plastique endommageable vers un comportement élastique endommageable «fragile ». Comme les constantes élastiques du matériau sont des caractéristiques intrinsèques, il est intuitif de proposer, d'abord, une évolution des caractéristiques matériaux relatives à la fonction seuil d'endommagement $\underline{Y}_{12}$. Pour un chargement en cisaillement quasi-pur (stratifié $[ \pm 45]_{\mathrm{S}}$ ), les constantes deviennent :

$$
\left\{\begin{array}{l}
G_{12}=G_{12}^{0}\left(1+F_{12}(\&)\right) \\
\underline{Y}_{12}=\sup _{\tau \leq t}\left(\sqrt{\frac{G_{12}}{2}}\left(2 \varepsilon_{12}^{e}\right)\right) \Rightarrow\left\{\begin{array}{l}
Y_{12 C}=\sqrt{\left(1+F_{12}(\&)\right)} Y_{12 C} \\
Y_{120}=\sqrt{\left(1+F_{12}(\&)\right) Y_{120}}
\end{array}\right. \\
d_{12}=\frac{\underline{Y}_{12}-Y_{120}}{Y_{12 C}}
\end{array}\right.
$$

Pour l'endommagement transversal, expérimentalement, on peut montrer que le coefficient de couplage $b$ demeure sensiblement constant et égal à sa valeur en statique et que les fonctions de viscosité sont très proches les unes des autres (cisaillement et transversales), de sorte qu'en première approximation, il est possible d'écrire [12]. Et enfin, nous émettons l'hypothèse supplémentaire que le taux d'énergie nécessaire pour rompre le stratifié (dans la direction transverse et/ou de cisaillement) est insensible à la vitesse de sollicitation : les caractéristiques critiques $\left(Y_{12 R}\right.$ et $\left.Y_{22 R}\right)$ sont par conséquent inchangées.

$$
Y_{22 C} \approx \sqrt{\left(1+F_{12}(\&)\right)} Y_{22 C} \text { et } Y_{220} \approx \sqrt{\left(1+F_{12}(\&)\right)} Y_{220}
$$

Nous avons donc imposé une évolution des constantes élastiques et d'endommagement afin de corroborer les états quasi statique et dynamique à une vitesse donnée. Il ne reste qu'à coupler la plasticité de la résine au modèle. Pour cela nous supposons que l'évolution des déformations plastiques est similaire à celle du 
cas statique. Cependant, elle est retardée puisque le matériau devient plus « rigide » : la contrainte d'écoulement initiale subit effectivement une évolution du même type que les modules d'élasticité :

$$
\left.R_{0}\right|_{\&}=\left(1+F_{R}(\&)\right) R_{0}
$$

En résumé, le comportement élasto-plastique endommageable visqueux de la résine est donné par les relations ${ }^{1}[14]$, [15] et [16].

$$
\left\{\begin{array}{l}
\sigma_{11} \\
\sigma_{22} \\
\sigma_{12} \\
\sigma_{13} \\
\sigma_{23}
\end{array}\right\}=\left(\begin{array}{ccccc}
C_{11} & v_{21}^{0} C_{11} & 0 & 0 & 0 \\
v_{21}^{0} C_{11} & C_{22}\left(\left(1-d_{22}\right)(a\rangle_{+}+\langle a\rangle_{-}\right) & 0 & 0 & 0 \\
0 & 0 & C_{12}\left(1-d_{12}\right) & 0 & 0 \\
0 & 0 & 0 & G_{13}^{0} & 0 \\
0 & 0 & 0 & 0 & G_{23}^{0}
\end{array}\right\}\left\{\begin{array}{l}
\varepsilon_{11}^{e} \\
\varepsilon_{22}^{e} \\
2 \varepsilon_{12}^{e} \\
2 \varepsilon_{13}^{e} \\
2 \varepsilon_{23}^{e}
\end{array}\right\}
$$

avec $C_{i j}=\left(C_{i j}^{0}\right)\left(1+\left.F_{i j}(\&)\right|_{i, j=1,2}\right.$ et $C_{i j}=\left.E_{i i}\right|_{i=j}$ ou $C_{i j}=\left.G_{i j}\right|_{i \neq j}$

$$
\left\{\begin{array}{l}
d_{12}=\frac{\left\langle\underline{Y}_{12}-Y_{120}\right\rangle_{+}}{Y_{12 C}} \text { si } d_{12,22}<1 \text { et } \underline{Y}_{22}<Y_{22 R} \\
d_{22}=\frac{\left\langle\underline{Y}_{12}-Y_{220}\right\rangle_{+}}{Y_{220}} \text { et } \underline{Y}_{12}<Y_{12 R} \text { sinon } d_{12,22}=1
\end{array}\right.
$$

$\operatorname{avec}\left\{\begin{array}{l}Y_{12 C}=\left(Y_{12 C}^{0}\right) \sqrt{\left(1+F_{12}(\&)\right)} \\ \left.Y_{120}=\left(Y_{120}^{0}\right) \sqrt{\left(1+F_{12}(\&)\right.}\right) \\ Y_{22 C}=\left(Y_{22 C}^{0}\right) \sqrt{\left(1+F_{12}(\&)\right)} \\ Y_{220}=\left(Y_{220}^{0}\right) \sqrt{\left(1+F_{12}(\&)\right)}\end{array}\right.$

1. L'exposant 0 caractérise les modules et les constantes issue de la loi élasto-plastique endommageable initiale (Gilletta, 1985), (Ladevèze, 1986), (Le Dantec, 1989), (Allix et al., 1990). 
$f_{P}(\widetilde{\sigma}, R)=\sqrt{\widetilde{\sigma}_{12} \widetilde{\sigma}_{12}+a^{2} \widetilde{\sigma}_{22} \widetilde{\sigma}_{22}}-R(p)-R_{0}$

avec $R_{0}=\left(R_{0}^{0}\right)\left(1+F_{R}\right)$

\section{Validations numériques des implémentations informatiques}

\subsection{Simulations numériques des essais de traction dynamique}

En premier lieu, il est nécessaire de vérifier que les comportements " primaires » théoriques sont bien représentés : pour cela, nous avons réalisé des validations dites élémentaires (Rozycki, 2000). Elles correspondent à la simulation des essais expérimentaux de traction ou de compression issus du cadre d'étude statique ou dynamique. Dans chacun des cas, l'éprouvette est représentée - puisque sa géométrie le permet - par un unique élément coque. Elle est encastrée d'un côté et de l'autre une fonction vitesse dépendante de la vitesse de sollicitation initiale est appliquée.

Les figures 6 et 7 en regard des figures 2 et 3 démontrent visuellement et quantitativement que l'approche développée semble être corrélée de façon satisfaisante avec l'expérience. Les pourcentages d'erreur commis sur le calcul des différentes constantes, par rapport à celles provenant des essais expérimentaux, sont globalement inférieurs à $10 \%$, ce qui confère à la modélisation visqueuse adoptée une certaine acuité pour ces types de sollicitation.

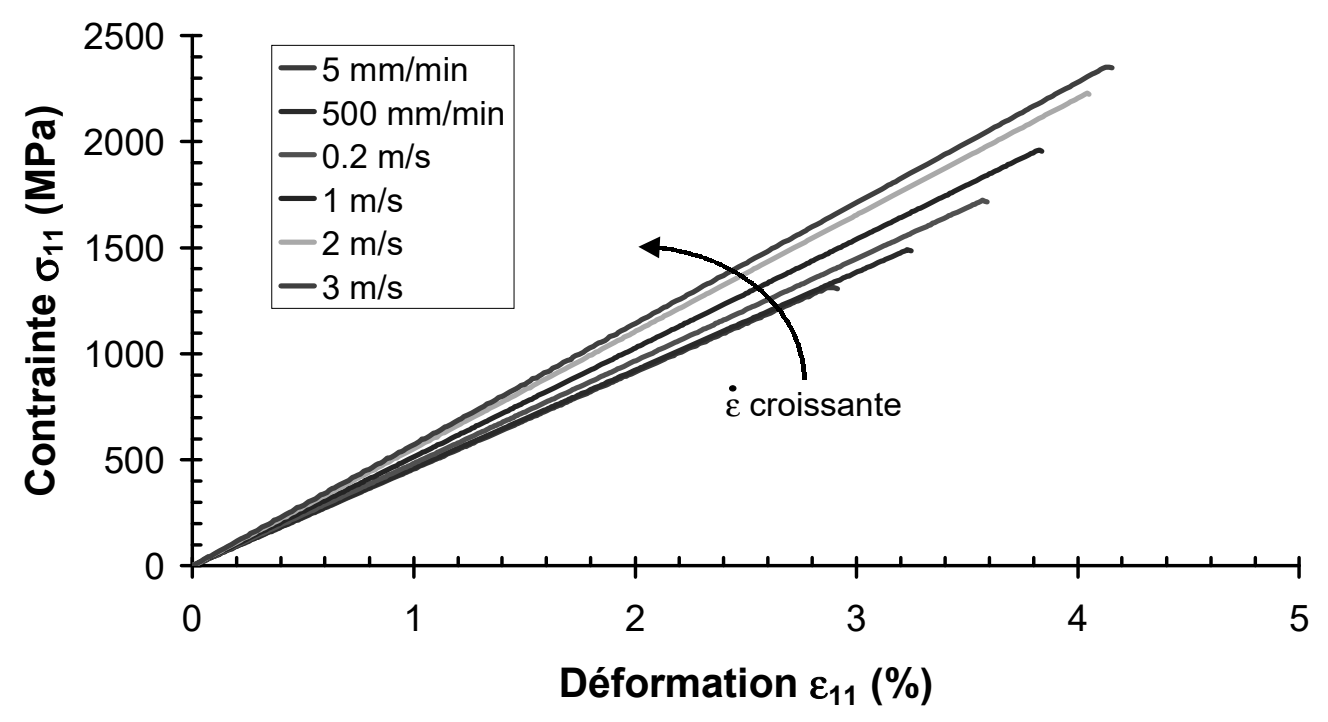

Figure 6. Tractions dynamiques numériques sur un stratifié $[0]_{4}$ 


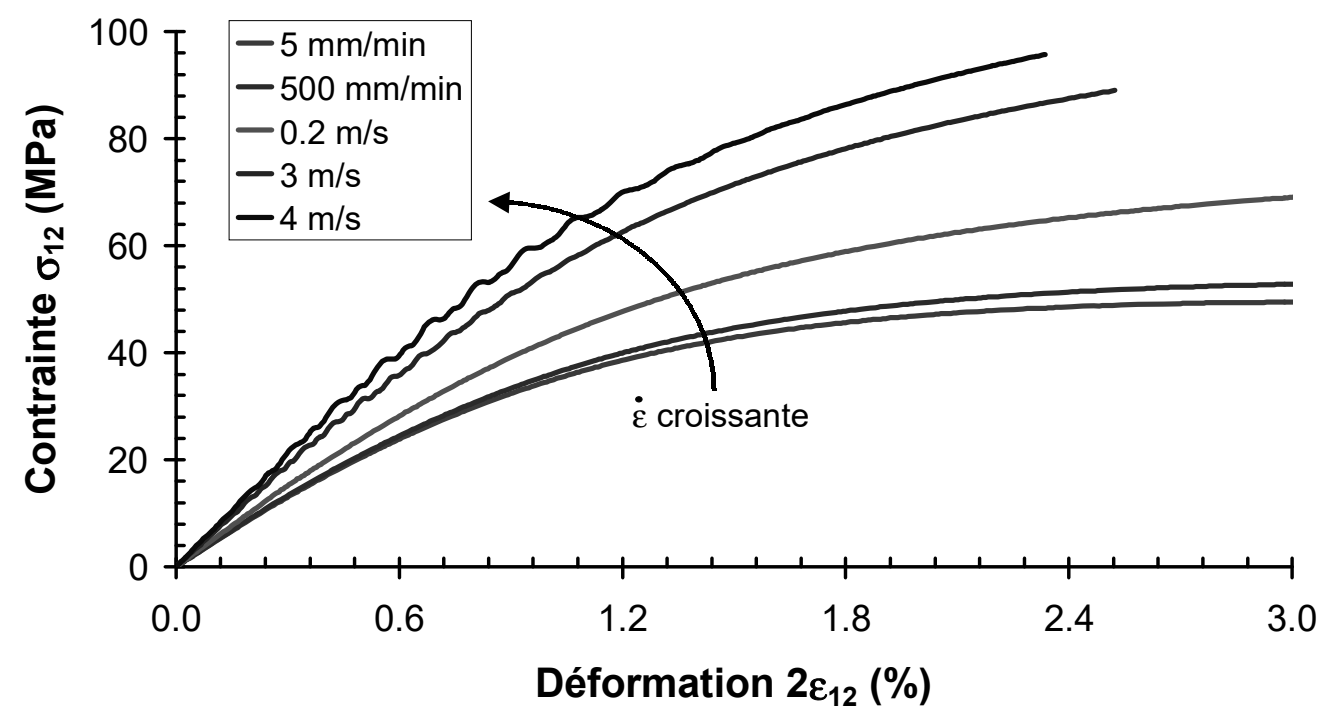

Figure 7. Tractions dynamiques numériques sur un stratifié $[ \pm 45]_{S}$

\subsection{Simulations d'essais d'impact sur des plaques}

Puisque la loi de comportement «visqueuse » semble être validée de manière satisfaisante pour les essais d'identification, nous avons engagé une campagne expérimentale à propos de sollicitations plus complexes afin de vérifier la fiabilité des développements: les essais expérimentaux se constituaient de flexions trois points dynamiques et d'impacts sur plaques de stratifications $\left[\begin{array}{llll}0_{2} & +45_{2} & -45_{2} & 90_{2}\end{array}\right]_{\mathrm{S}}$ et $\left[\begin{array}{lll}+45_{2} & -45_{2}\end{array}\right]_{2 \mathrm{~s}}$. Nous présentons dans cette partie quelques premiers résultats (Rozycki, 2002) issus des dépouillements d'un impact sur une plaque $\left[0_{2}+45_{2}-45_{2}\right.$ $\left.90_{2}\right]_{\mathrm{s}}$ par une bille sphérique qui, couplée à un chariot, propose une masse de 26.5 $\mathrm{kg}$ tombant à une vitesse au moment de l'impact de $4 \mathrm{~m} / \mathrm{s}$.

Sur la figure 8, nous comparons l'évolution expérimentale et numérique de l'effort appliqué en fonction du temps. Les premières constatations tendent à démontrer que l'intégration de la sensibilité à la vitesse de déformation est une précaution non-négligeable puisqu'elle permet de corriger la rigidité de la structure de façon adéquate : la vitesse de déformation moyenne observée au point d'impact est de l'ordre de $100 \mathrm{~s}^{-1}$ (figure 9); la description temporelle de l'effort semble à l'aide de l'optimisation de la loi comportementale plus satisfaisante que celle proposée par la modélisation «quasi statique». On retrouve effectivement le premier effort maximal supporté par la plaque aux alentours de $1.5 \mathrm{~ms}$.

Cependant, la figure 8 met en évidence que le taux d'effort maximum appliqué à la plaque reste toujours inférieur à l'expérimental bien que la description temporelle soit satisfaisante : en effet, nous avons observé au cours des essais l'apparition de délaminage entre certains plis (aire hachurée sur la figure 8). Or les modélisations numériques que nous avons adoptées ne tiennent pas compte de ce phénomène; nous supposons par conséquent que cette différence de taux d'effort provient d'une 
dégradation («numérique ») brutale plutôt que progressive de la structure. L'état d'endommagement se répartit de façon prompte et intégrale suivant l'épaisseur de la coque alors qu'expérimentalement l'apparition de délaminage autorise la conservation d'une certaine intégrité et rigidité de la structure : les plis, bien qu'ils soient délaminés par endroits, interagissent toujours avec l'ensemble de la structure. Des études sont actuellement en cours afin d'appliquer une méthodologie développée au sein du laboratoire pour valider cette hypothèse (Walrick, 1999), (Walrick et al., 2000), (Walrick et al., 2002).

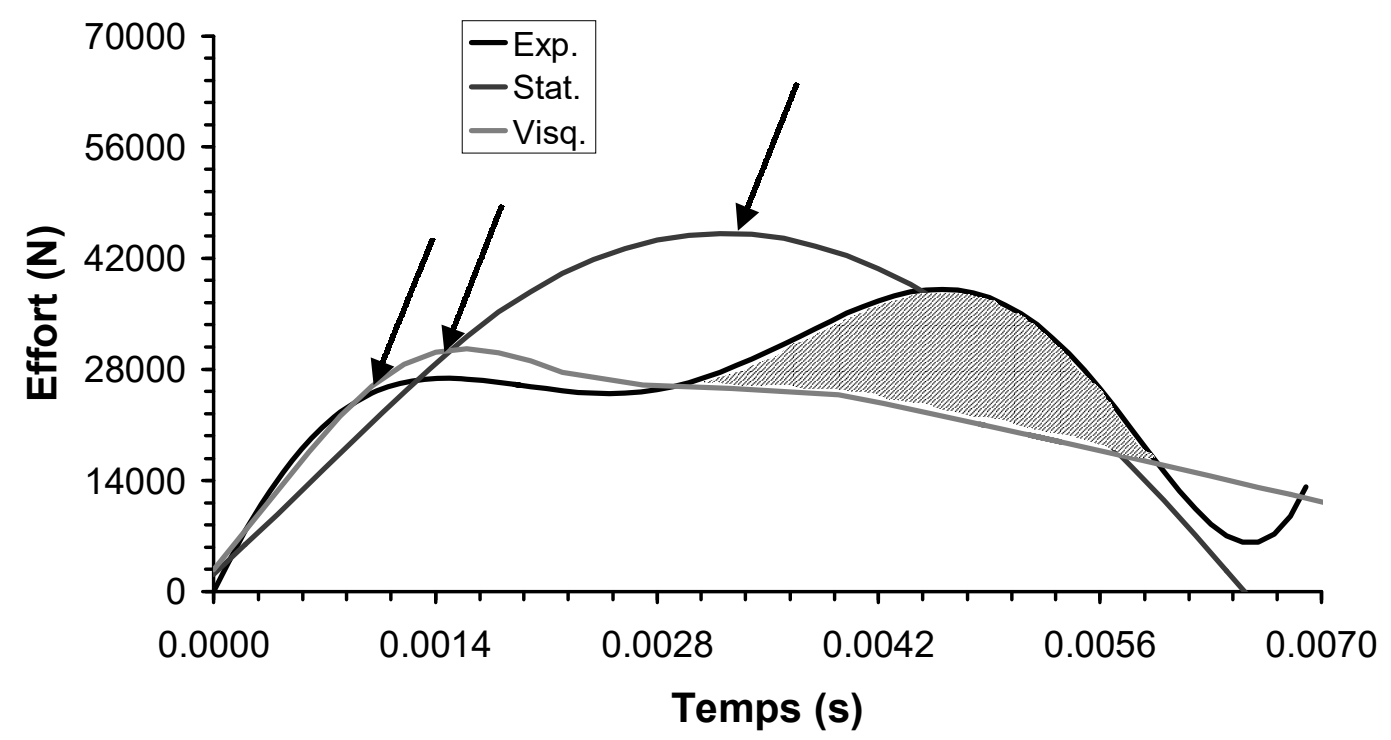

Figure 8. Comparaisons expérimental/numérique pour un impact
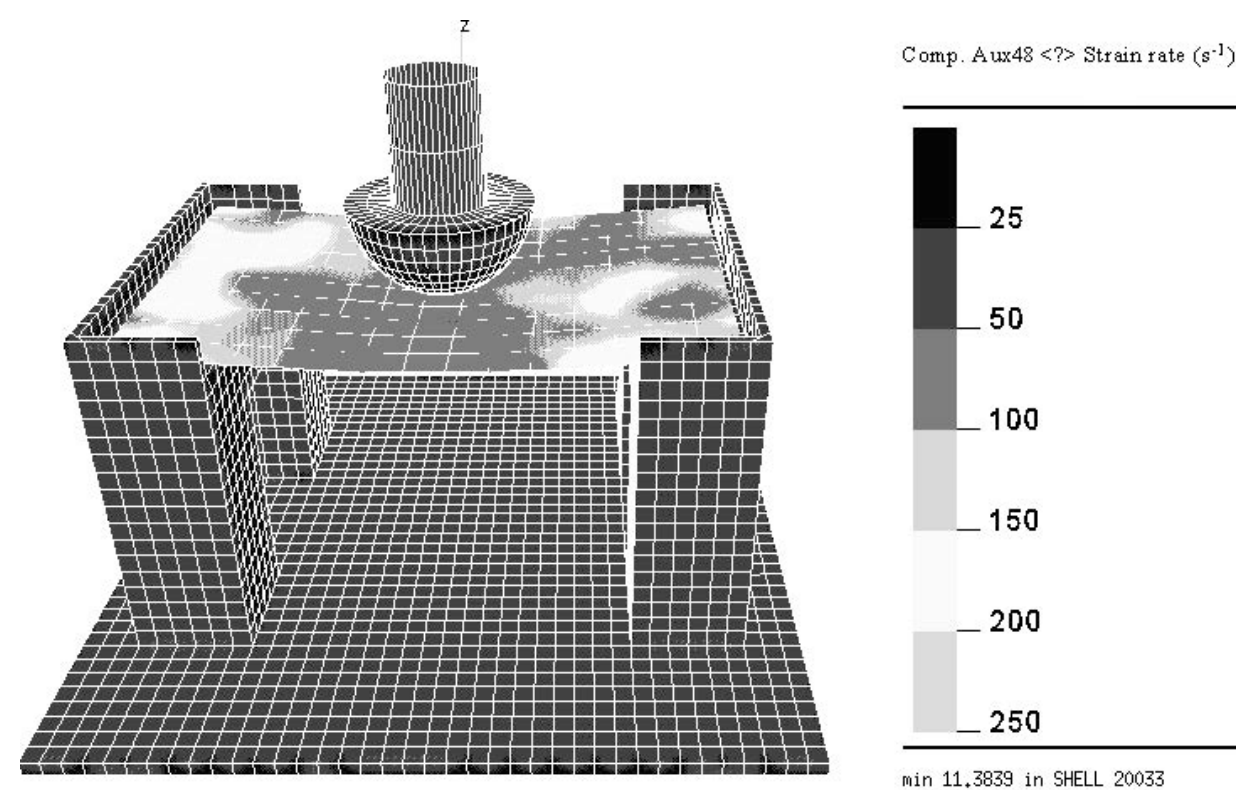

Figure 9. Contour numérique de la vitesse de déformation 


\section{Conclusions et perspectives}

Les quelques résultats exposés, nous permettent de mettre en évidence une certaine pertinence de l'approche originale adoptée. En effet, que ce soit pour les validations élémentaires ou pour les validations complexes, les développements théoriques et informatiques aboutissent à une prise en compte satisfaisante de l'influence de la vitesse de déformation sur le comportement du matériau. De plus, à la méthodologie expérimentale de caractérisation de la loi élasto-plastique endommageable, nous avons adjoint un complément permettant l'identification de manière simple, rapide et facile à mettre en œuvre des lois d'évolution pour la viscosité, ce qui conserve les atouts principaux de cette méthodologie.

L'ensemble de cette étude a été transposée aussi aux composites tissés, pour notamment des études sur des composites carbone/époxy dans le cadre d'un projet européen (HICAS, High Velocity Impact of Composite Aircraft Structures, CECE DG XII BRITE-EURAM Project BE 96-4238, 1998). Des conclusions similaires ont été mises en évidence tant pour la méthodologie expérimentale de caractérisation proposée que pour le concept théorique visqueux développé (Rozycki, 2000) (Johnson et al., 2001)

Ces résultats nous encouragent par conséquent à poursuivre et à affiner les travaux, notamment en proposant une description plus appropriée intégrant les phénomènes d'élévation de température. De plus, d'autres études sont en cours afin d'intégrer les phénomènes majeurs de délaminage.

\section{Remerciements}

Nous tenons à remercier, tout d'abord, Messieurs E. Haug et X. Ni de la société ESI Software ainsi que l'ANRT qui ont permis la réalisation de ces travaux de thèse dans le cadre d'une convention CIFRE. Nous adressons ensuite nos plus vifs remerciements à l'ONERA centre de Lille, et plus particulièrement à Messieurs E. Deltombe et D. Dormegnie, pour nous avoir soutenus et permis de réaliser l'ensemble des essais expérimentaux. Enfin, nous remercions le CNRS et la région Nord-Pas de Calais pour leur soutien permanent.

\section{Bibliographie}

Allix O., Deü J.F., Ladevèze P., «A delay-damage meso-model for the prediction of localisation and fracture of laminates subjected to high-rate loading. », In: ECCM'99 Proc. European Conference on Computational Mechanics, Munich, Germany, 31 August - 3 September, 1999.

Allix O., Ladevèze P., Le Dantec E., « Modélisation de l'endommagement du pli élémentaire des composites stratifiés », Journées Nationales sur les Composites 7, Lyon, Editions Pluralis, 1990, p. 715-724. 
Chaboche J.L., Formalisme général des lois de comportements: applications aux métaux et polymères. Introduction à la mécanique des polymères, Editions G'sell C. \& Haudin J.M., Nancy, 1995, p. 119-140.

Coutellier D., Gauthier C., Ravalard Y., Ni X. \& Haug E., « Un élément multimatériaux pour l'étude du comportement en crash des structures stratifiées: synthèse et perspectives », Revue Européenne des Eléments Finis, Vol. 7, n 1/2/3, 1998, p. 177-192.

Coutellier, D., Rozycki, P., «New functionalities for PAMCRASH ${ }^{\mathrm{TM}}$ multilayered multimaterial element », Composites Part A Applied Science and Manufacturing, Vol. 31, 2000, p. 841-851.

Gauthier C., Contribution à la modélisation du comportement en crash des structures stratifiées métal/composite, Développement d'un élément de coque multicouches multimatériaux, Thèse de Doctorat, Université de Valenciennes et du Hainaut-Cambrésis, 1996.

Gilletta de Saint Joseph D., Composite 2D : modélisation mécanique et identification de la couche élémentaire, Thèse de Doctorat, Université Pierre et Marie Curie, Paris 6, 1985.

Johnson A.F., Pickket A.K. et Rozycki P., «Computational methods for predicting impact damage in composite structures », Composite Science and Technology, Vol-61 (15), 2001, p. 2183-2192.

Ladevèze P., "Sur la mécanique de l'endommagement des composites », Journées Nationales sur les Composites 5, Paris, Editions Pluralis, 1986, p. 667-683.

Le Dantec E., Contribution à la modélisation du comportement mécanique des composites stratifiés, Thèse de Doctorat, Université Pierre et Marie Curie, Paris 6, 1989.

Lemaitre J. et Chaboche J.L, Mécanique des matériaux solides, Editions Dunos, Paris, 1985, chapitre 1 : p. 26-31, chapitre 4 : p. 145-148.

Pam Crash ${ }^{\mathrm{TM}}$, Solver Notes Manual \& Solver Reference Manual. ESI Group Software product company, Rungis, 2000.

Rozycki P, Contribution au développement de lois de comportement pour matériaux composites soumis à l'impact, Thèse de Doctorat, Université de Valenciennes et du Hainaut-Cambrésis, 2000.

Rozycki P., Validations numériques de la loi élasto-plastique endommageable visqueuse Essais de flexion trois points dynamiques et impact de plaques, rapport interne de l’Université de Valenciennes, à paraître en 2002.

Walrick J.C., Contribution au développement d'une nouvelle méthodologie pour l'étude du délaminage dans les structures stratifiées composites : application à l'impact basse vitesse, Thèse de Doctorat, Université de Valenciennes et du Hainaut-Cambrésis, 1999.

Walrick J.C., Coutellier D. \& Geoffroy P., « Méthodologie pour une prévision du délaminage dans la modélisation de structures stratifiées composites. », Mécanique \& Industries, 2000, Vol. 3, p. 259-266.

Walrick J.C., Coutellier D., \& Geoffroy P., «Development of a new methodology for delamination detection in laminated structures. », Integrated Design and Manufacturing in Mechanical Engineering'2000, In Chedmail, Cognet, Fortin, Mascle \& Pegna Kluwer Academic Publishers Editions, à paraître en mars 2002. 